\title{
Analysis of the effect of infection prevention nursing on drainage of malignant pleural effusion with indwelling central venous catheter
}

\author{
Yu Zhao ${ }^{1}$, Lijin Zhong ${ }^{2}$, Qinna Mao ${ }^{1}$, Guifei Huang ${ }^{3}$, Hualiang Zhang ${ }^{1}$, Xia Xuan ${ }^{4}$ \\ ${ }^{1}$ Department of Critical Care Medicine, Zhuji Affiliated Hospital of Shaoxing University, Zhuji, China; ${ }^{2}$ Operating Room, Zhuji Maternal and Child \\ Health Hospital, Zhuji, China; ${ }^{3}$ Department of Cardiothoracic Surgery, Zhuji Affiliated Hospital of Shaoxing University, Zhuji, China; ${ }^{4}$ Department \\ of Critical Care Medicine, Zhuji Affiliated Hospital of Shaoxing University, Zhuji, China \\ Contributions: (I) Conception and design: Y Zhao, L Zhong; (II) Administrative support: None; (III) Provision of study materials or patients: Q Mao; \\ (IV) Collection and assembly of data: H Zhang, G Huang; (V) Data analysis and interpretation: X Xia; (VI) Manuscript writing: All authors; (VII) \\ Final approval of manuscript: All authors. \\ Correspondence to: Xia Xuan. Zhuji City Chinese Medicine Hospital Critical Care Medicine, 521, Huandong Street East Second Road, Zhuji 311800, \\ China. Email: 573589215@qq.com.
}

\begin{abstract}
Background: The aim of this study was to explore the effect of infection prevention care on the drainage of malignant pleural effusion (MPE) by indwelling Central venous catheterization (CVC).

Methods: From January 2016 to January 2018, 128 patients at our hospital who needed indwelling CVC for drainage of MPE were randomly divided into an infection prevention group and a conventional group. The corresponding nursing plan was given to compare the 2 groups in several measures, including nursing effect and complications.

Results: After intervention, the total effective rate of the infection prevention group was $96.88 \%$, while the total effective rate of the conventional group was $87.50 \%$, which was a statistically significant difference $(\mathrm{P}<0.05)$. The disappearance time of pleural effusion, catheter indwelling time, and length of stay in the infection prevention group were significantly lower than those in the conventional group $(\mathrm{P}<0.05)$. The incidence of infection and the total incidence of all complications in the infection prevention group were significantly lower than those in the conventional group $(\mathrm{P}<0.05)$. The proportion of the number of cases with Karnofsky Performance Scale (KPS) $\leq 10$ in the infection prevention group was significantly lower than that in the conventional group $(\mathrm{P}<0.05)$.
\end{abstract}

Conclusions: As infection prevention care significantly improves clinical efficacy and reduces the occurrence of complications, it uses in clinic is warranted.

Keywords! Infection preventive care; central venous catheterization (CVC); malignant pleural effusion (MPE)

Submitted Feb 03, 2021. Accepted for publication Mar 23, 2021.

doi: $10.21037 /$ apm-21-532

View this article at: http://dx.doi.org/10.21037/apm-21-532

\section{Introduction}

Malignant pleural effusion (MPE) is a type of pleural effusion characterized by the transfer of malignant cells to the pleura or other causes of pleural effusion (1). MPE is a common complication of malignant tumors, with an incidence of $15 \%$ in cancer patients $(2,3)$, which usually indicates that the disease has reached the advanced stage, and is associated with high morbidity and mortality, along with reduced treatment options. MPE is most commonly found in lung cancer, followed by breast cancer (4), with the common symptoms being dyspnea, cough, and chest pain.

Central venous catheterization $(\mathrm{CVC})$ is a process which 
Table 1 General information of 128 patients with MPE

\begin{tabular}{|c|c|c|c|c|}
\hline Project & Infection prevention group & Conventional group & $t / \chi^{2}$ & $\mathrm{P}$ \\
\hline Sex (male/ female) & $38 / 26$ & $40 / 24$ & 0.1313 & 0.7171 \\
\hline $\begin{array}{l}\text { Type of malignant tumor (lung cancer/breast } \\
\text { cancer) }\end{array}$ & $35 / 29$ & $33 / 31$ & 0.1225 & 0.7232 \\
\hline Volume of malignant pleural effusion $(\mathrm{mL})$ & $160.79 \pm 20.18$ & $158.44 \pm 22.56$ & 0.6211 & 0.5357 \\
\hline
\end{tabular}

MPE, malignant pleural effusion.

uses a multifunctional device, in which a long, soft, thin and hollow catheter is placed in a large vein (blood vessel), which can be used to monitor hemodynamic indicators, intravenous drugs, liquids, blood products, and external tube nutrition. In contrast to intravenous catheterization through the hand or arm, in CVC, the middle line is longer, the tube diameter is thicker, the blood flow is faster, and the blood flow is heavier. This prevents irritating drugs and hypertonic or viscous liquids from damaging the peripheral blood vessels and reduces the occurrence of chemical phlebitis $(5,6)$. For MPE, CVC puncture drainage is also a common practice in clinical practice.

The method is mainly used because the traditional puncture and aspiration fluid requires multiple extractions to reduce the effect of pleural effusion, which is easily affected by the patient's physical strength, fluid extraction time, and other factors. Closed drainage of the chest cavity will cause larger skin wounds, and thus CVC is applied. The drainage method involves no skin incision, little trauma to the chest wall, less damage to internal organs, and good drainage effect. Although indwelling CVC can be beneficial to MPE patients, CVC is always an invasive procedure, and entails difficulty in avoiding infection risk, with catheterrelated-blood-stream infection (CR-BSI) being the greatest risk. The incidence of CR-BSI is currently on the rise, and it has become a nosocomial infection that cannot be ignored. How to control CVC-related infections has become an important issue in clinical research, and effective preventative care against $\mathrm{CVC}$ is are critical to reducing concurrent infections in patients. Consequently, to explore the impact of infection prevention care on CVC-related infections, this study examined 128 patients who needed indwelling CVC for puncture and drainage of MPE.

We present the following article in accordance with the CONSORT reporting checklist (available at http:// dx.doi.org/10.21037/apm-21-532).

\section{Methods}

\section{General information}

A total of 128 patients with MPE who needed indwelling CVC and drainage from January 2016 to January 2018 at Zhuji People's Hospital and Zhuji Traditional Chinese Medicine Hospital in Zhejiang Province were included in the study. Patients were divided into an infection prevention group and a conventional group according to the nursing plan and the patients' baseline data. Patient characteristics are summarized in Table 1.

The inclusion criteria for patients were as follows: (I) meeting the diagnostic criteria of malignant tumors in the "Chinese Standards for the Diagnosis and Treatment of Common Malignant Tumors"; (II) all cancer cells discovered by pleural fluid exfoliate cytology and diagnosed as MPE as confirmed by pleural ultrasound or computed tomography (CT); (III) informed consent and voluntary enrollment. The exclusion criteria were as follows: (I) heart, liver, and kidney failure; (II) with diseases such as extensive atelectasis and fixed mediastinum.

All procedures performed in this study involving human participants were in accordance with the Declaration of Helsinki (as revised in 2013). The study was approved by Zhuji People's Hospital (NO.2020-10) and informed consent was taken from all the patients.

\section{Nursing plan}

The interventions of the conventional group were as follows:

(I) Preoperative and psychological care: the physical and psychological conditions of MPE cases were assessed and given corresponding psychological interventions based on the evaluation results to enhance the patient's self-confidence in recovery 
and promote the patient's active cooperation with treatment.

(II) Pre-puncture care: the nurses were strictly required to perform aseptic operations throughout the entire process, which included using iodophor to disinfect the puncture site 3 times and covering it with a sterile dressing.

(III) Intraoperative care: the temperature of the room was adjusted appropriately to ensure patient comfort and closely monitor the patient's physical signs during the puncture process. After the puncture was completed, the nurse assisted in connecting the disposable drainage bag, turned off the catheter switch in time when separating the catheter to prevent gas entry, disinfected the puncture site, and fixed the drainage tube with a transparent film.

(IV) Postoperative care: performing blood drawing, transfusion, blood transfusion, or other related operations at the CVC site were prohibited. Targeted postural care for patients with intubation was conducted in different areas. For example, patients with internal cervical and subclavian vein catheters would have their heads tilted to the contralateral position and patients with femoral vein catheters would be placed in a supine position. Patients were informed that the drainage bag should be placed properly and drained. The placement was $40-50 \mathrm{~cm}$ below the puncture point, and attention was paid to protecting the drainage bag so as to prevent the drainage bag from being pulled out.

In addition to the measures implemented in the conventional group, the infection prevention group received the following infection prevention care:

(I) Maximization of the sterile barrier: while using headwear, masks, sterile gloves, etc., nurses were required to treat the puncture site of the patient. The site of puncture was fully exposed and the patient's whole body was covered with a sterile treatment towel.

(II) Enhanced disinfection: the CVC material was disinfected with a nonalcoholic disinfectant.

(III) Connection and fixation of the drainage tube: Strengthen fixation of patients with thin body to prevent the drainage tube from folding, sliding, twisting. Dressings were selected according to the specific conditions of the patients. For patients with high fever and sweating, gauze dressing was the first choice, while for patients with thinner bodies, the fixation was strengthened. The gauze dressing was replaced at a frequency of at least 48 hours/change, and the change frequency for the transparent film was at least 7 days/change. If the dressing was wet, curled or loose, it was replaced in timely fashion.

(IV) Observation of the puncture point: the puncture point surrounding the skin was closely observed each day, the condition of the puncture point and surrounding skin was evaluated, and any signs of inflammation such as redness, swelling, oozing, etc. were immediately reported to the doctor.

(V) Blockage prevention: if the drainage fluid was thick and turbid, the lumen was first flushed with $30 \mathrm{~mL}$ of normal saline when clamping the tube, and the tube was sealed with heparin saline to prevent blockage.

(VI) Drainage tube management: the drainage tube was clamped when the patient was resting at night, and the drainage was opened when the patient was awake during the day. If the drainage tube was open for a long time, the drainage bag was replaced regularly, usually every $3-5$ days to prevent retrograde infection.

\section{Observation indicators}

The observation indicators are listed below.

Efficacy judgment: chest imaging examination was performed 3 months after discharge to observe the absorption of MPE.

Significant effect: When MPE is completely absorbed, there is no obvious pleural hypertrophy or adhesion, the costophrenic angle became dull, or pleural thickness was $<0.5 \mathrm{~cm}$, the nursing effect is significant.

Effective: care was considered effective when MPE was partially absorbed and the pleural hypertrophy and adhesion were moderate.

Ineffective: care was considered ineffective when MPE absorption time was $>2$ months, there was thickening of the pleura $>1 \mathrm{~cm}$, or an encapsulated effusion had formed.

The total effective rate was calculated as follows: total effective rate $=($ significantly effective + effective $) / 64$.

The Karnofsky Performance Scale (KPS) score was used to assess the patient's quality of life 3 months after discharge. Significant improvement: KPS increased by at least 20 score between pre-treatment and post-treatment; Improvement: KPS increased by at least 10 score from before to after treatment; Stable: No change in KPS before and after treatment; Decline: Decline in KPS of at least 10 score between before and after treatment. The occurrence 
Table 2 Comparison of general indicators

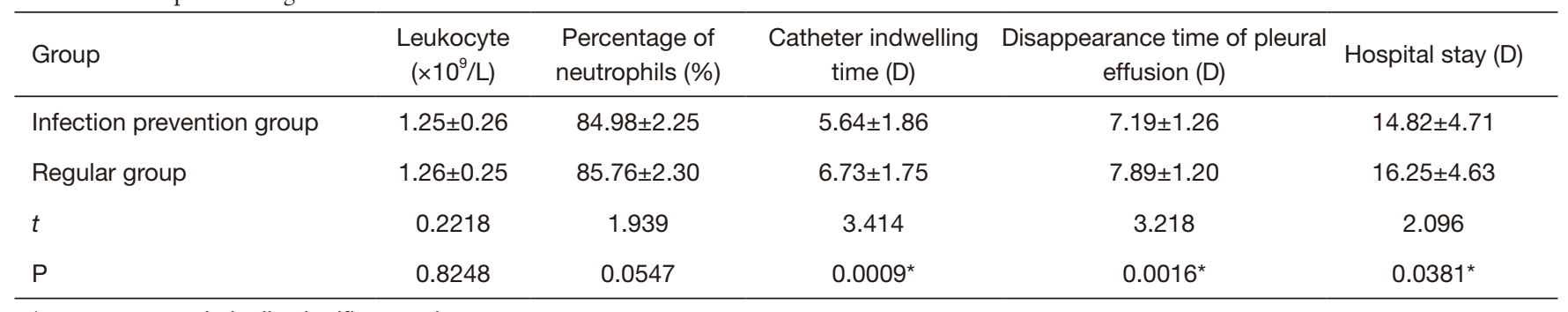

*, represent statistically significant values.

Table 3 Comparison of complications

\begin{tabular}{|c|c|c|c|c|c|c|}
\hline Group & Infection (n) & Blocked catheter (n) & Catheter falls off (n) & Thrombus (n) & Pneumothorax (n) & Total incidence (\%) \\
\hline$\chi^{2}$ & 4.800 & 0.3413 & 1.008 & 0.2081 & 1.008 & 4.137 \\
\hline
\end{tabular}

*, represent statistically significant values.

Table 4 Evaluation of quality of life

\begin{tabular}{lcccc}
\hline Group & $\begin{array}{c}\mathrm{KPS} \geq 20 \\
{[\mathrm{n}(\%)]}\end{array}$ & $\begin{array}{c}\mathrm{KPS} \geq 10 \\
{[\mathrm{n}(\%)]}\end{array}$ & $\begin{array}{c}\mathrm{KPS}=0 \\
{[\mathrm{n}(\%)]}\end{array}$ & $\begin{array}{c}\mathrm{KPS} \leq 10 \\
{[\mathrm{n}(\%)]}\end{array}$ \\
\hline $\begin{array}{l}\text { Infection prevention } \\
\text { group }\end{array}$ & 48 & 12 & 3 & 1 \\
$\begin{array}{l}\text { Conventional group } \\
\chi^{2}\end{array}$ & 42 & 10 & 5 & 7 \\
$\mathrm{P}$ & 1.347 & 0.2196 & 0.5333 & 4.800 \\
\hline
\end{tabular}

*, represent statistically significant values. KPS, Karnofsky Performance Scale.

of complications, catheter indwelling time, disappearance time of pleural effusion during the patient's hospitalization, and the patient's white blood cell level before discharge were recorded.

\section{Statistical processing}

SPSS v.20.0 (IBM Corp., Armonk, NY, USA) software package was used for data analysis. Measurement data are expressed as $\bar{x}$ $\pm s$, and the comparison of the means between two groups was performed by $t$ test. Count data are expressed by percentages, and the comparison was performed by $\chi^{2}$ test. A P value $<0.05$ was considered statistically significant.

\section{Results}

\section{Comparison of general indicators}

After intervention, the disappearance time of pleural effusion, catheter indwelling time, and length of stay in the infection prevention group were significantly lower than those in the conventional group $(\mathrm{P}<0.05)$ (Table 2$)$.

\section{Complications}

After intervention, the incidence of infection and the total incidence of all complications in the infection prevention group were significantly lower than those in the conventional group $(\mathrm{P}<0.05)$ (Table 3).

\section{Evaluation of quality of life}

After intervention, the proportion of cases with KPS $\leq 10$ in the infection prevention group was significantly lower than that in the routine group $(\mathrm{P}<0.05)$ (Table 4).

\section{Comparison of nursing effects}

In the infection prevention group, 40 cases were effective, 22 cases were effective, 2 cases were ineffective, and the 
Table 5 Comparison of nursing effects between the two groups

\begin{tabular}{lcccc}
\hline \multirow{2}{*}{ Group } & \multicolumn{3}{c}{ Markedly } & \multicolumn{3}{c}{ Effective } & Invalid Total effective \\
& effective (n) & (n) & (n) & rate (\%) \\
\hline $\begin{array}{l}\text { Infection prevention } \\
\text { group }\end{array}$ & 40 & 22 & 2 & 96.88 \\
Conventional group & 36 & 20 & 8 & 87.50 \\
$\chi^{2}$ & & & & 3.905 \\
$\mathrm{P}$ & & & & $0.0481^{*}$ \\
\hline
\end{tabular}

*, represent statistically significant values.

total effective rate was $96.88 \%$. In the conventional group, 36 cases were effective, 20 cases were effective, 8 cases were ineffective, and the total effective rate was $87.50 \%$. The total effective rate of the infection prevention group was significantly higher than that of the routine group $(\mathrm{P}<0.05)$ (Table 5).

\section{Discussion}

CR-BSI ranks third in hospital infections and accounts for $20-30 \%$ of hospital-acquired sepsis (7). There are approximately 200,000 cases of CR-BSI in the United States each year, and up to 4,000 deaths due to CR-BSI each year $(8,9)$. CR-BSI has myriad causes, and $60 \%$ of CR-BSI cases are related to the extraluminal colonization of skin-derived microorganisms during or within a few days after insertion of the CVC. Many studies have shown that coagulasenegative staphylococci need to attach to human skin. Oxygen bacteria are also the most common pathogens of CR-BSI $(10,11)$. In addition, the CVC interface and the cultivation of pathogenic bacteria in the CVC cavity are also common ways of infection. The general CVC interface is a spiral design with uneven surfaces, and if disinfection is not thorough, pathogens are likely to colonize the recesses and cause infection. When the cap is connected with the scalp needle, the scalp needle may accidentally be exposed to the air, and the in-and-out movement of the needle may cause pathogens to invade the catheter from the joint, and enter the body with the infusion of liquid, causing infection (12).

At present, many strategies for the prevention of CRBSI exist, including maximizing sterile barriers and CVC care. A prospective randomized study conducted by Raad et al. (13) compared maximum sterile barrier preventative measures (e.g., sterile protective clothing and gloves, headwear, masks and large sterile drapes) and conventional preventative measures (sterile gloves and small drape).
The enhanced sterile barrier reduced the risk of CR-BSI from $0.5 / 1,000$ to $0.02 / 1,000$ (13). CVC care consists of reasonable selection and replacement of auxiliary materials, care of catheter joints and injection surface, and other measures. Placing a dressing on the catheter insertion site can protect the area from external contamination, and can fix the device. Generally, a transparent film is used because it has the advantages of easy fixation and direct observation of the puncture point, but it is transparent and has poor water absorption capacity. For patients with hyperhidrosis, sweat beads may be wrapped in the film. The accumulation of sweat reduces the viscosity of the film and curls the film. This can expose the puncture point, leaving it vulnerable to contamination, so the choice of dressing should be based according to the specific situation of the patient. At present, there is no consensus in the literature concerning dressing selection and replacement frequency. In the 2006 version of the American Infusion Therapy and Nursing Practice Standards, it was recommended that the replacement frequency of gauze dressing be at least 48 hours/change and the replacement frequency of sterile transparent film be at least 7 days/change; furthermore, it was recommended that if the dressing is wet, curled, or loose, it should immediately be replaced (14). For the care of CVC joints and injection surface, Chinese researchers recommend that alcohol swabs should be used to thoroughly disinfect the interface and heparin cap screw. The disinfection time should last at least 30 seconds before the next step is begun (6). In addition, impregnating CVCs with antibacterial drugs is another effective method to prevent CR-BSI. Maki et al. (15) reported that the colonization rate with uncoated catheters was twice as high as that of polyurethanes CVCs with external surfaces coated with chlorhexidine and silver sulfonamide, resulting in a 4-times reduction in the possibility of bloodstream infection. It can be seen from the above studies that various nursing measures can reduce the risk of CR-BSI. As nursing staff play an essential role in preventing CR-BSI, it is crucial to develop effective nursing methods in preventing and controlling the occurrence of CR-BSI and improve the quality of nursing.

A study by Webster et al. (16) showed that the risk of CR-BSI was greatly reduced by strengthening nursing interventions in infection prevention. The incidence of catheter infection in infection prevention care was only $2.86 \%$. In this study, on the basis of routine care, we strengthened the care of infection prevention for MPE patients, which mainly included maximizing the sterile barrier, strengthening disinfection, selecting the appropriate 
dressing according to the patient's specific situation, and closely monitoring the puncture point. The results show that these nursing practices produced significant clinical benefits. After infectious nursing intervention, the disappearance time of pleural effusion, catheter indwelling time, and length of stay in the infection prevention group were significantly lower than those of the conventional group $(\mathrm{P}<0.05)$, demonstrating that infection prevention care can help drainage of pleural effusion. The time of effusion disappearance, catheter indwelling time, and length of hospital stay were shorter, suggesting that the curative effect was superior. In addition, the incidence of infection and the total incidence of all complications in the infection prevention group were significantly lower than those in the conventional group $(\mathrm{P}<0.05)$, suggesting that infection prevention care can greatly reduce the risk of infection and other complications during drainage. Three months after discharge, the total effective rate of the infection prevention group was $96.88 \%$, which was significantly higher than the total effective rate of $87.50 \%$ of the conventional group $(\mathrm{P}<0.05)$. This further confirmed that the infection prevention care effect was good and could significantly improve the long-term clinical efficacy. The proportion of cases with KPS $\leq 10$ in the infection prevention group was significantly lower than that in the conventional group $(\mathrm{P}<0.05)$, and fewer people in the infection prevention group had a lower quality of life, indicating that after treatment, the quality of life of most patients in the infection prevention group was improved.

In summary, infection prevention care significantly improves clinical efficacy and reduces complications, and is thus a valuable tool in clinical practice.

\section{Acknowledgments}

Funding: None.

\section{Footnote}

Reporting Checklist: The authors have completed the CONSORT reporting checklist. Available at http:// dx.doi.org/10.21037/apm-21-532

Data Sharing Statement: Available at http://dx.doi. org/10.21037/apm-21-532

Conflicts of Interest: All authors have completed the ICMJE uniform disclosure form (available at http://dx.doi. org/10.21037/apm-21-532). The authors have no conflicts of interest to declare.

Ethical Statement: The authors are accountable for all aspects of the work in ensuring that questions related to the accuracy or integrity of any part of the work are appropriately investigated and resolved. All procedures performed in this study involving human participants were in accordance with the Declaration of Helsinki (as revised in 2013). The study was approved by Zhuji People's Hospital (NO.2020-10) and informed consent was taken from all the patients.

Open Access Statement: This is an Open Access article distributed in accordance with the Creative Commons Attribution-NonCommercial-NoDerivs 4.0 International License (CC BY-NC-ND 4.0), which permits the noncommercial replication and distribution of the article with the strict proviso that no changes or edits are made and the original work is properly cited (including links to both the formal publication through the relevant DOI and the license). See: https://creativecommons.org/licenses/by-nc-nd/4.0/.

\section{References}

1. Psallidas I, Kalomenidis I, Porcel JM, R et al. Malignant pleural effusion: from bench to bedside. Eur Respir Rev 2016;25:189-98.

2. Bibby AC, Dorn P, Psallidas I, et al. ERS/EACTS statement on the management of malignant pleural effusions. Eur J Cardiothorac Surg 2019;55:116-32.

3. Skok K, Hladnik G, Grm A, et al. Malignant Pleural Effusion and Its Current Management: A Review. Medicina (Kaunas) 2019;55:490.

4. Feller-Kopman DJ, Reddy CB, DeCamp MM, et al. Management of Malignant Pleural Effusions. An Official ATS/STS/STR Clinical Practice Guideline. Am J Respir Crit Care Med 2018;198:839-49.

5. Fahy B, Sockrider M. Central Venous Catheter. Am J Respir Crit Care Med 2019;199:P21-2.

6. Safety Committee of Japanese Society of Anesthesiologists. Practical guide for safe central venous catheterization and management 2017. J Anesth 2020;34:167-86.

7. Pandit P, Sahni AK, Grover N, et al. Catheter-related blood stream infections: prevalence, risk factors and antimicrobial resistance pattern. Med J Armed Forces India 2021;77:38-45.

8. Thompson S, Wiebe N, Klarenbach S, et al. Catheter- 
related blood stream infections in hemodialysis patients: a prospective cohort study. BMC Nephrol 2017;18:357.

9. Rello J, Ochagavia A, Sabanes E, et al. Evaluation of outcome of intravenous catheter-related infections in critically ill patients. Am J Respir Crit Care Med 2000;162:1027-30.

10. Safdar N, Maki DG. The pathogenesis of catheter-related bloodstream infection with noncuffed short-term central venous catheters. Intensive Care Med 2004;30:62-7.

11. Maki DG. Infection caused by intravascular devices: pathogenesis, strategies for prevention. In: Maki DG, editor. Improving Catheter Site Care. London: Royal Society of Medicine Services, 1991:3-27.

12. Zeng P, Tao J, Yang Y, et al. Central venous catheter indwelling and related infection analysis. Chinese Journal of Practical Pediatrics 2003;18:743-4.

Cite this article as: Zhao Y, Zhong L, Mao Q, Huang G, Zhang H, Xia X. Analysis of the effect of infection prevention nursing on drainage of malignant pleural effusion with indwelling central venous catheter. Ann Palliat Med 2021;10(3):3379-3385. doi: 10.21037/apm-21-532
13. Raad II, Hohn DC, Gilbreath BJ, et al. Prevention of central venous catheter-related infections by using maximal sterile barrier precautions during insertion. Infect Control Hosp Epidemiol 1994;15:231-8.

14. Gorski LA. The 2016 Infusion Therapy Standards of Practice. Home Healthc Now 2017;35:10-8.

15. Maki DG, Stolz SM, Wheeler S, et al. Prevention of central venous catheter-related bloodstream infection by use of an antiseptic-impregnated catheter. A randomized, controlled trial. Ann Intern Med 1997;127:257-66.

16. Webster J, Osborne S, Rickard CM, et al. Clinicallyindicated replacement versus routine replacement of peripheral venous catheters. Cochrane Database Syst Rev 2019;1:CD007798.

(English Language Editor: J. Gray) 